pathology and improve the treatment of hydrophobia.

It will, however, be sufficiently evident, from the experiments already cited, that saliva is capable of exerting a very marked influence upon the brain and nervous system. The spasm, convulsion, and coma, which were consequent upon the introduction of saliva into the arteries and veins, are conclusive proofs of its activity; whilst the absence of all such symptoms on the injection of other animal fluids into the circulatory system, demonstrates that not to any physical or mechanical influence, but to a peculiar property inherent in itself, is saliva indebted for the manifestation of its physiological action.

That saliva is capable of producing local as well as general nervous excitement, will be learnt from those of the experiments which relate to its effects when introduced into the stomach; whence we may infer, that one of its purposes in assisting the function of digestion, consists in affording a natural and necessary stimulus to the organ employed in the digestive process.

I shall now proceed to consider the further use of saliva in reference to digestion.

\section{PATHOLOGY OF SUDDEN DEATH.}

\section{To the Editor of The LANCET.}

SrR,-I beg to transmit the following cases as contributions to the pathology of sudden death. Of nine cases, it will be seen that in six there was organic disease of the heart; in two, congestion of the brain; and in one, the only assignable cause of death was fatal syncope. I remain, Sir, your obedient servant,

Islington, March 12, 1842.

\section{Case I. - Sudden Death from Disease of the Heart.}

John Charter, ætat. 58, admitted as a patient into the Islington Infirmary, Sept. 19, 1835; he had been employed for several years as an ostler, and was a man of intemperate habits. He had generally enjoyed good health, till a few weeks before his admission, when he was attacked with an affection of the chest, for which he received no medical advice. The symptoms on his admission were as follows:- $\mathrm{He}$ complains of pain and tightness in his chest with difficulty of breathing; he lies indifferently on either side ; is very restless in bed, and is obliged to have his head supported in consequence of the urgent dyspnoea; tongue clean; pulse moderate, but hard and wiry; abdomen swelled; legs anasarcous; face pale; urine rather scanty; scrotum dropsical; bowels not much confined; little appetite; some thirst. The auscultation of the chest proved that the heart and lungs were dis* eased; the impulse of the former being very strong, but without any morbid sound; and the latter giving evidence of inflammation of the air-tubes by sonorous and sibilant rhonchi heard on both sides of the chest. This man was ordered to lie in bed with his head elevated, to drink toast and water, or acidu. lated drinks, and to use a moderate diet. He was also ordered a blister to be applied to the chest, and the following medicines:-

\section{R Jalap powder, $3 \mathrm{j}$;}

Bitartrate of potash, $3 \mathrm{ij}, \mathrm{ft}$. The powder to be taken three times a-day.

$\mathrm{R}$ Solution of iodide of potassium, m. xx; Mint water, $3 \mathrm{j}$. ft. The draught to be taken every four hours.

These medicines caused him to pass a great quantity of watery evacuations, and afforded him some relief; they were continued, with some alteration of the quantities, until Oct. 2nd, when the abdomen was so enormously distended with fluid, that paracentesis was deemed advisable, and this ope. ration was accordingly performed. Three gallons of straw-coloured serum were removed, and he expressed himself very much relieved. His health began gradually to improve, he rose from his bed, and was able to walk about the ward, and he appeared rapidly to be approaching to his ordinary state of health. But on the 23rd of Decem. ber, twelve weeks after tapping, and more than three months after his admission, he expired suddenly while walking to the watercloset.

On examining the body, the heart was found much increased in size; the left ven. tricle was hypertrophied and dilated, but there was no disease of the valves. The lungs were congested, and the bronchial tubes thickened and inflamed, and filled with frothy mucus. The brain was not examined.

CASE II.-Sudden Death from Disease of the Heart.

James Miles, ætat. 55, admitted as a patient into the Islington Infirmary, Jan. 15 , 1840. The impulse of the heart was very strong, and heard over a large space; the lips and face were livid; the legs anasar. cous. He was ordered the following :-

$\mathrm{R}$ Tincture of digitalis, $\mathrm{m} . \mathrm{x}$; Infusion of senna, 3x ; Epsom salts, 3ij. The draught to be taken every four hours.

R. Bitartrate of potass, $3 \mathrm{ij}$;

Nitrate of potass, gr. $\mathrm{x}$. The powder to be taken twice a-day.

This man died suddenly four days aftel his admission.

\section{Post-mortem Examination.}

Chest.-The heart was enlarged to abou twice its natural size; the parietes of bot ventricles were bypertrophied, but thoi 
cavities were of the natural size. The pulmonic semilunar, the mitral, and the tricuspid valves were healthy, but the aortic semilunar valves were rigid and cartilaginous. The dorta was much dilated to the distance of about four inches from its origin, and the pouch so formed presented internally a large quantity of calcareous particles, arranged in plates and scattered in granules. This osseous deposition was traced at intervals along the whole course of the thoracic and abdominal aorta as far as its bifurcation. The left lung was healthy, and the bronchial tubes did not present any marked morbid appearance. The right lung was adherent to the parietes of the chest by strong fibrous bands; the lower part of this lung was hepatised and very friable, the middle portion was engorged, the upper part healthy; in other words, the inferior and middle portions of this lung exhibited the first and second stages of pneumonia. The existence of pneumonia as a consequence or an accompaniment of diseased cardiac valves, has been pointed out by many authors, and I have seen several cases of the kind in $m y$ own experience.

Abdomen.-The liver was large, and presented the nutmeg appearance; the other viscera of the abdomen were healthy. 'The brain was not examined.

In this case there were preparations evidently made for an aneurism of the aorta, had not death put an end to the process. The existence of osseous matter in the lining membrane of the arteries, although common enough in advanced age, is by no means frequently found in persons so little advanced in years as the subject of the present account.

In the two cases just detailed, death must be attributed to a spasmodic affection of the heart causing a sudden cessation of its action; a result which was anticipated from the great increase in size of that organ. But in the next case, which is a very curious one, although the heart and lungs were, no doubt, originally in fault, the immediate cause of death was a fatal and profuse hæmorrhage from the mucous surfaces.

\section{CASE III.-Sudden Death from Profuse Hamorrhage.}

John Carter, atat. 78, was taking down some article from a shelf when he was suddenly seized with a profuse vomiting of blood, and died in a ferv minutes. I attended immediately afterwards, and found a large quantity of blood on the floor, and about a quart in a basin; it appeared of a florid colour. The mouth, nostrils, and face of the man were covered with blood.

Post-mortem Examination Twenty-four Hours after Death.

On removing the sternum the right lung appeared very large, and extended over to the left side ; it was of a dark colour, and did not collapse on the removal of the sternum. On its anterior and inferior surface a dark, blackish patch appeared, about the size of a hen's egg : on cutting into which, it presented a dark, bloody, congested appearance, not, however, bounded by any definite border, and resembling the disease called by Laennec apoplexy of the lungs. Other similar congestions were seen in other parts of the right lung. The left lung was extremely small, collapsed, and impermeable to air, except at a very few points near the edge; there were strong adhesions on this side between the costal and pulmonary portions of the pleura, and the substance of the lung was soft and friable. On cutting into this lung, the edges were found permeable to air ; the lower part was hard and fleshy, while the upper and middle portions appeared broken down and ulcerated, and the arteries were found crossing the cavities in the form of strong, hard cords. No phthisical tubercles, however, were seen in any part. At the point where the left pulmonary artery entered the lung, the passage was found to be impervious; and on further examination it was found to be closed up by the growth of a fleshy, dense substance around its walls. The heart appeared externally to be somewhat enlarged; the two auricles were found healthy, as was also the right ventricle; but the left ventricle was dilated and hypertrophied, the dilatation being slight, but the hypertrophy considerable; the septum ventriculorum very much thickened. The aortic semilunar valves were found hardened by a copious deposition of calcareous matter; there seemed, however, sufficient room for the passage of the blood. The aorta was found to be degenerated; patches of albuminous "matter were secreted along its course, with atheromatous deposits situated below the lining membrane. The trachea was examined and found healthy; but on accidentally opening the vesophagus, blood was seen to issue from the wound, which circumstance led me to examine the stomach very carefully. This organ was large, and constricted in the middle. On opening it, it was found filled with an enormous quantity of dark, grumous blood, mixed up with mucus and half-digested food. On cleaning the stomach, a layer of gelatinous bloody matter was found lying on its mucous membrane, and was removed with some little difficulty. The mucous membrane was found in the stomach to be generally red and injected. No vessel, however, could be found ruptured, though the coeliac axis and its arteries were found degenerated in the same manner as the aorta mentioned above. I considered this case as one of hæmateme. sis; but Dr. Copland, who saw it reported in the "Medical Gazette," and has noticed it in his Dictionary, article Hæmorrhage, thinks, with more probability, that it was really one of hæmorthage from the trachea 
and bronchial tubes, some of the blood $\}$ and emphysematous at their edges. The making its way into the stomach and causing other viscera presented no remarkable mor. vomiting, and thus leading to an appearance bid appearance.

of hæmatemesis. This explanation certainly accounts for a fact which perplexed me exceedingly, for while the blood on the floor of the room was quite florid, that in the stomach was darkened by the gastric juice.

In the three next cases which $I$ am about to detail, the disease of the heart, which was the immediate cause of death, was complicated with congestive or inflammatory disease of the brain. This connection of cardiac with cerebral disease is very properly insisted upon by several modern writers; and I may mention that in my own practice I have found disease of the heart accompanied by sanguineous apoplexy, meningitis, and ramolissement.

CAse IV_-Sudden Death from Disease of

the Heart, attended with Meningitis.

Sarah New, ætat. 66, was brought into the infirmary, Feb. 22, 1841, in a state of collapse, with weak, fluttering pulse, livid lips, and pale countenance. A glass of brandy with hot water was ordered to be administered immediately, after which she rallied a little, and expressed herself better. A mixture containing aromatic spirit of ammonia and compound tincture of cardamoms was also prepared and sent, but before it arrived she had expired. From the time of her seizure till her death, which took place in about an hour and a half, she was perfectly sensible, complaining of no pain, but of excessive exhaustion. A post-mortem examination of the body was made thirtyeight hours after death, when the following appearances were observed :-

Head.-The scalp, skull, and dura mater were healthy. The arachnoid membrane was highly inflamed, and its vessels injected to an extreme degree of minuteness; a large quantity of pale, transparent, serous fluid was found between its layers, and escaped upon making an ivcision into the membrane. The brain was very soft in its consistence; the nerves were torn asunder by the slightest tension; the ventricles contained but little fluid. It is a circumstance worthy of notice. that notwithstanding this serious cerebrai disease, there was no evidence, before death, of any affection of the brain or its membranes.

Chest.-The heart was found to be diseased, it was enlarged much beyond the natural size, and, on examining its interior, it was found that the walls of both ventricles were much thickened, and the carneæ columnæ increased both in size and number, but the cavities of the veniticles were rather below than above the natural size. This was a case, therefore, of simple hypertrophy. The valves were healthy, and the aorta was of its natural texture and diameter. The lungs were roluminous, crepitating throughout,

\section{Case V.-Siudden Death from Disease of the} Heart, attended with Meningitis.

John Grant, ætat. 49, a navigator, a strong, muscular, and well-formed man, of intemperate habits, living in Islington, had obtained a letter for the Islington Dispen. sary, on account of cough and shortness of breath. He was not seen by the physician, either from some misunderstanding, or because he did not suppose, from the repre. sentations of the relatives, that it was a case of sufficient importance to require visiting. Indeed, it does not appear that his wife her. self thought her husband in any immediate danger, for on the very morning of his death he had expressed himself better. While his wife was gone to the dispensary to request the physicran to visit her husband, he was sitting up in bed, and asked for some beer, which was given to him by a woman in the room, immediately after drinking which be fell back in the bed and expired. I was sent for and came immediately, but he was dead before I arrived. A coroner's inquest sat upon the body, and returned a verdict of " natural death." A post-mortem examination had been previously made by order of the coroner, when the following appearances were observed :-

Head.-The scalp and skull presented no peculiar appearance. The vessels of the dura mater were injected. The arachnoid membrane was highly inflamed and thick. ened, and a considerable quantity of thin serous fluid was found between its layers. The pia mater was healthy. The substance of the brain was firm, healthy, and of it: natural colour, and although cut in evers direction exhibited no signs either of abscess congestion, or sanguineous effusion.

Chest.-The lungs were very much in creased in size, and in a high state of con gestion. The bronchial tubes exhibited the marks of intense and long-standing inflam mation; they were much thickened, and th lining membrane was of a deep-red colour marked with red longitudinal strix, an filled with a large quantity of frothy an muco-purulent matter. The heart was ver much enlarged, being about twice its naturo size, and very fat. The right ventricle con tained some fibrinous clots; the left vel tricle some half-coagulated blood. The cavit of each ventricle, especially the left, wa enormously distended; the walls were about the usual thickness, and the carne: columna were of ordinary size : the cast therefore, was one of dilatation of the hear with hypertrophy. Had the parietes of th ventricles been thinner than in the natur state the disease would have been simple d latation, which is but rarely seen. Th 
valves were bealthy, and appeared to offer no obstruction to the current of the blood.

Abdomen.-The liver was congested, and of a dark colour. The stomach was healthy, and nearly empty. All the other viscera were quite healthy.

\section{Case VI.-Sudden Death from Disease of} the Heart, attended with Sanguineous Effusion into the Brain.

Jane Harwood, æatat. 68, an inmate of the Islington workhouse, had long suffered from difficulty of breathing and palpitations of the heart. The action of the heart was accelerated, its impulse very strong, and heard over a greater space than is natural; pulse very feeble and quick. She had been several times attacked with violent fits of dyspnoea and faintness, which had been relieved by diffusible stimulants, and mustard-poultices to the calves of the legs. She had also taken small doses of tincture of digitalis, tincture of opium, and gentle purgatives, with considerable benefit. As I had long anticipated sudden dissolution in this case, she was ordered to remain in the infirmary, to be kept perfectly quiet, and to have a light nutritious diet. But having been for some time previously in a comparatively good state of health, she was suddenly seized, on the evening of the 31st of May, 1841, with violent pain in the left side, with difficulty of breathing. When I visited her, very shortly after the attack, I found her in articulo mortis, with very rapid and feeble pulse, cold extremities, convulsive movements of the mouth, pale and bloodless lips, eyes fixed and halfclosed. Mustard poultices were immediately applied to the region of the heart and the calves of the legs, and hot bricks to the soles of the feet. The following medicine was prescribed :-

$\mathrm{IX}$ Compound tincture of lavender, $\mathrm{m} . \mathrm{x}$; Mint water, $z^{\mathrm{i}}$;

but before it could be sent she expired.

\section{Post-mortem Examination Thirty-six Hours after Death.}

Heud.-The scalp, skull, and membranes of the brain were healthy. But on examining the left ventricle that cavity was found filled with an enormous coagulum of blood, which not only filled that ventricle, but had passed through the third ventricle, and occupied a great part of the right ventricle.

Chest.-There were some slight old adhesions in both lungs, and there was a considerable quantity of thin serous fluid effused into the cavities of the pleuræe on both sides. The lungs were voluminous, crepitant throughout, and emphysematons at their edges and on their surfaces. The bronchial tubes were of a dark-red colour on their internal surface, which was marked by longitudinal purple striæ, and contained a small quantity of frothy mucus. The pericardium contained a small quantity of thin serous fluid. The heart externally appeared about one-half larger than its natural size ; on being opened it was found that the cavity of the left ventricle was somewhat diminished in diameter, but not to a great extent, while the walls were enormonsly thickened, and the carnea columna of great size. The aorta and pulmonary artery were quite free from disease, and their valves were healthy.

Aldomen.-The liver, which was of its natural size, presented a veined and granular appearance, both externally and internally, resembling what is called the nutmeg liver. The kidneys were below the natural size; externally they presented several vesicles of about the size of a bean, and containing serum; internally the glandular tissue was absorbed, and its place in great measure supplied by fatty and fibrous matter.

In the three following cases of sudden death no disease of the heart could be detected.

\section{Case VII.-Sudden Death from Cerebral Congestion.}

Sarah Holt, ætat. 17, an inmate of the Islington workhouse, had been in the infirmary for some time on account of suppression of the menses. She was a stout, healthylooking girl, of a florid complexion. She took the medicines usually prescribed for amenorrhoea, and became much better, but still the menstrual discharge was not restored. She left the infirmary, apparently cured, on Monday, March 1, 1842. On Tuesday she pursued her usual avocations, and about four o'clock in the afternoon engaged in a fight with another girl. After eating her supper she went to bed at the usual hour, and while in bed she complained of pain and uneasiness to the persons in the same ward; but her complaints were not of such a character as to induce her to ask for medical assistance. The next morning she was found dead in her bed, with her face downwards. A coroner's inquest was held upon the body, and as there were no circumstances of suspicion attaching to the case, a verdict of " natural death" was returned. After the inquest a post-mortem examination was made, when the following appearances were observed :-

Head.-The vessels of the scalp were turgid with blood; and on removing the skull the brain was found to be in a high state of congestion, the veins and sinuses being greatly distended with fluid blood. Beyond this general congestion of its vessels the brain presented no morbid appearance, and no extravasation could be detected in any part.

Chest.-The heart was perfectly healthy, as were likewise the lungs, with the exception of some old adhesions between the two surfaces of the pleura.

Abdomen.-The stomach, intestines, and liver were all healthy. The bladder was 
empty. The uterus, which might bave been supposed to exhibit some morbid appearance, was small, of healthy structure, and its cavity empty.

\section{Case VIII.-Sudden Death from Cerebral Congestion.}

The infant child of Hannah L., in the Islington infirmary, was found by the nurse, in the middle of the day, lying dead at a short distance from the mother, who was awake. The infant was three days old, and illegitimate. The only remark made by the mother, on being informed of the infant being dead, was, " $\mathrm{Oh}$, is it !" and she neither offered any explanation of the manner of its death, although she must have been awake at the time, nor did she testify surprise or regret at the event. No peculiar circumstances attended the labour, which was a natural and easy one, and the infant was perfectly healthy. On examination the body presented numerous appearances of lividity on the upper as well as the under part, the livid marks being most perceptible about the head. The hands were clenched; the tongue protruded beyond the jaws. Around the neck there were impressions which to the parties who examined the body appeared to have been caused by a ligature. Under these circumstances it was judged expedient to give intimation of the occurrence to the coroner, and an inquest was accordingly held upon the body. No evidence whatever was adduced, either to criminate the mother or to account for the death by natural causes. A post-mortem examination was ordered, when the following appearances were observed:-

Head. The vessels of the scalp were congested; the dura mater was adherent not only to the fontanelles, but also to a great part of the inner table of the cranial bones. The sinuses and veins of the brain were universally turgid with blood, but there was no extravasation; and the substance of the brain was healthy, only presenting the softness peculiar to that organ in new-born children.

Neck.-The integuments were carefully dissected from the region of the supposed ligature, when it was found that there were no marks on the muscles of the neck, nor on the arteries or veins, corresponding to the external impressions; and no doubt was, therefore, left on the mind that the lines observed upon the skin were produced by natural causes.

Chest.-The lungs were healthy; the heart was also healthy, containing a little fluid blood. The other viscera were quite heal thy. A verdict of " natural death" was returned.

The above appears to me a most important case. The infant, who only an hour or two ago appeared to every person who saw it in the enjoyment of perfect health, dies without a groan and without a struggle, in the middle of the day, by the side of its mother, who was herself lying awake at the time, in a large room which contained no person be" sides herself. When informed of her infant's death she exhibits no surprise or emotion. There are suspicious appearances on the in. fant's body, which correspond to those popu. larly known as attendant upon strangulation. I feel happy in the reflection that the post. mortem examination enabled my father and myself to obliterate the suspicions at first entertained, even by ourselves, and to save the unhappy mother from the danger of a criminal prosecution.

\section{Case IX.-Sudden Death from Syncope.}

Mrs. R., aged 43, a lady of great talents and accomplishments, residing in Islington, had been under the care of Dr. Babington for some weeks, in consequence of a pleuritic effusion into the right side of the chest. She was not considered in any danger, and her husband and son had both gone to town as usual, leaving her at home with the servant. Between three and four in the afternoon of Friday, Mrarch 4, she was in bed, and had occasion to use the night-pan, and soon after passing a motion she became suddenly very ill and faint; and though I and another medical gentleman were sent for and attended immediately, she expired before our arrival.

The next day, at five o'clock, Dr. Babington made the post-mortem examination, at which I was present. I then learned that Dr. B. had treated the case as one of effu. sion into the right side of the chest, for which he had prescribed a blister, with small doses of blue-pill, and tincture of opium, in combination with tonics. He had stated to the lady's husband that the disease was curable. The post-mortem examination will prove that Dr. B.'s prognosis and diagnosis were, at the time he made them, perfectly correct, although the former was falsified by some mysterious agency, the operation of which no human science could foresee or prevent.

Chest.-On inserting a trocar between the ribs on the right side, a straw-coloured fluid was poured out. Nearly two quarts of this fluid were found between the costal and pulmonary portions of the pleura on this side, the pleural surfaces being thickened and connected together by numerous delicate filamentous bands which traversed the fluid. The lung of this side was very much com pressed, and the two lower lobes nearly obliterated. The left lung was quite healthy. The heart was pushed very far to the left side. This organ was flabby, the walls of about the ordinary thickness, the cavities slightly dilated, the valves all healthy. The blood in the heart, as well as in the other parts, was fluid. It is remarkable that there was no indication externally to denote effusion into the chest: both sides appeared to be of equal size, and no prominence was observed in the intercostal 
spaces. On percussion, however, the right side was quite dull, the left gave healthy sound.

Abdomen.-The liver was excessively enlarged, extending over to the left side, and downwards to the iliac region: its structure, however, only presented the appearance of great congestion. The spleen was congested and friable. The inner membrane of the stomach presented a dark red colour and corrugated appearance, the results either of congestion or chronic inflammation. The kidneys, uterus, and intestines, were quite healthy.

The brain was not examined.

The cause of death in this case is involved in considerable obscurity; the fatal result cannot be attributed to the effusion into the chest, because empyema is by no means an incurable disease, or if fatal, it is never rapidly so ; and as the other lung and the heart were healthy, the risk of sudden dissolution could not be reasonably anticipated. Dr. Babington attributes the death of the patient (and for want of any better explanation, I am induced to coincide in his opinion) to the fact that she became exhausted from the evacuation of the bowels, and sunk into a fatal syncope. Considering the large quantity of fluid contained in the right side of the chest, and pressing upon the heart, and the enormously enlarged liver also pressing upwards upon that organ, together with the enfeebled state of the patient, it is probable that the action of the heart might have become suddenly arrested by the slight excitement referred to, and a fatal termination induced.

ILLUSTRATIONS OF THE

\section{PATHOLOGY, DIAGNOSIS, AND TREATMENT}

$\mathrm{OF}$

\section{OPHTHALMIC AFFECTIONS.}

By Edward O. Hocken, M.D., M.R.C.S.L., F.R.M.C.S., \&c.

$$
\text { Part XIII.* }
$$

THE AMAUROSES.

Wishing to illustrate completely the subject of the varieties, causes, incipient and confirmed symptoms, and the effects of treatment in the amauroses, depending on hyperæmia of the general visual nervous system, I will narrate the few remaining but condensed cases which I possess.

At that period of life when the catamenia ought to occur, or at a time shortly afterwards, if they fail to be secreted, or having appeared are again suppressed by causes which derange the general health, different constitutional diseases may be established, leading to different results; either the gene-

* Continued from vol. i., p. 639, 1841-2.

No. 972. ral powers may sink, the vascular system become comparatively empty and impoverished in its contents, with those other derangements in the economy constituting chlorosis; or, on the contrary, the vascular may become absolutely or relatively fullthe one with an exuberance of power, the other with a comparative deficiency in vital energy. Amaurosis may occur, and does occur comparatively often, in either of these two last constitutional conditions; relative plethora is, however, by far the most common. The following case appears to me to well illustrate this last form :-

Complete Amaurosis of the Right Eye ; imperfection of Vision in the Left; dull, oppressive Headach; Non-appearance of the Menses.

Mary Hallett, sixteen years of age, was admitted into the West of England Eye Infirmary, on the 21 st of May, 1841. She states that for a series of years she always enjoyed uninterrupted good health, and that her habitual employments were simply confined to domestic affairs. Her sight had been failing for the last six months, especially in the right eye, in which organ she had been completely blind for the three months immediately preceding her admission; whilst the left has been gradually getting worse since that period. During the whole time of the existence of these symptoms she had been subject to very severe, deep-seated, but dull and oppressive headach, especially affecting: the right side. She states that when about six years old she became blind in both eyes, and continued in this condition for twelve months, at the expiration of which period she was perfectly cured by bloodletting, which was employed locally, and that she has remained perfectly well ever since, until the commencement of her present attack.

Symptoms.-As regards her present symptoms, her countenance appears flushed, bloatel, and full-looking; conjunctivæ rather more vascular than natural; both pupils widely dilated, and very sluggish in their movements, being but little sensible of the influence of light; the globes generally, and their component textures, appear healthy, She complains of complete loss of vision in the right eye, being quite unable to distinguish light from darkness, or day from night, with that organ; and of great imperfection of sight, with cloudy, misty, and deranged appearance of objects viewed with the left eye. At present her headach is very severe; she describes it as deep-seated, oppressive, but very severe, and as affecting the right side especially. She has never suffered from intolerance of light. Her tongue appears natural; her pulse is quick and weak, but full ; her bowels regular; but she had never menstruated. 